\title{
Reconstruction of Super Resolution Images with Correlation Based Sub-Pixel Registration
}

\author{
Tuba Saltürk ${ }^{1}$, and Abdullah $\mathrm{Bal}^{2}$
}

\begin{abstract}
The accuracy of super resolution application depends on the performance of registration, interpolation and restoration steps. In this stage, the rate of sub-pixel accuracy, which processed in registration step, holds an important place for providing the success of super resolution application. In this paper, correlation based subpixel registration methods are handled and how these methods influence over the accuracy of the super resolution application is explored. In this scope, registrations achieved by correlation measure criteria combined with Bilinear, New Edge Directed Interpolation (NEDI) and Cubic Spline methods one by one and these registration results are utilized in super resolution application. Experimental results show that, using correlation combined with Cubic Spline interpolation obtain higher performance than correlation combined with other interpolation methods in a super resolution application.
\end{abstract}

Keywords - Image registration; interpolation; super resolution; image enhancements.

\section{INTRODUCTION}

A super resolution application produces high resolution (HR) image using low resolution (LR) image or images. A process of super resolution image reconstruction consists of registration, interpolation and restoration steps. Using registration for improving resolution has been attracting a lot of interest in this research area since Tsai and Huang [1] proposed the idea for the first time in 1984.

The quality of a high resolution image, which reconstructed by a super resolution algorithm is directly related to the registration method used in the application. Image registration is a process of transforming different images into the same coordinate axis and it becomes more successful while more sub-pixel accuracy obtained. When there is an integer shift between the LR images, it means that there is no extra information. So the super resolution images produced from these LR images is the zoomed version of one LR image.

In this paper, Bilinear, NEDI, Cubic Spline interpolation methods are utilized to determine the sub-pixels accurately. Among these methods, Cubic Spline with correlation measure criteria increases the accuracy of registration method and improves the performance of super resolution application more than other interpolation methods.

\section{Sub PIXEL REgISTRATION}

A transform function to yield the geometrical registration between images is defined to represent the images obtained

\footnotetext{
${ }^{1}$ Department of Informatics, 2Department of Electronics and Communication, Yıldı Technical University, Istanbul
}

from different sources or same source but different times in the same coordinate plane. It is aimed to bring this function to maximum or minimum value according to the selected registration method. In a super resolution application, the objective is to reach HR image pixels as constants. It is also assumed that there is translation between LR images. The amount of shift is found by the registration methods.

The measure criteria may vary in registration methods. In this scope, the most common measure criterias are defined as cross correlation [2], phase correlation, mutual information and sum of squared difference. The interpolation method used in the registration takes an important place, since it creates new pixels and these pixels change the amount of translation.

In this paper, horizontal and vertical shifted LR images are registrated by correlation [3-4] measure criteria combined with Bilinear, NEDI, and Cubic Spline interpolation. In order to get HR image, each result obtained from interpolation methods are combined with the Projection Onto Convex Sets (POCS) super resolution method. The HR images are finally evaluated by SSIM and PSNR metrics.

\section{A. Bilinear Interpolation}

Bilinear Interpolation is a resampling method that uses the 4 nearest neighboring values in the diagonal axis of missing value. In the images, missing pixel is estimated by taking the average of 4 neighboring pixels of LR image. This process continues till the every missing pixel is filled. The resultant image has smooth translations. The formula of this method is as follow.

Assume that, $\mathbf{X}$ is the LR image and $\mathbf{Y}_{\mathbf{k n} . \mathbf{l}}$ is the missing pixel of interpolated image.

$$
Y_{k, l}=\frac{X_{i, j}+X_{i+1, j}+X_{i, j+1}+X_{i+1, j+1}}{4}
$$

\section{B. New Edge Directed Interpolation (NEDI)}

This method is arisen from the idea of using covariance coefficients of LR images for adapting interpolation at the HR image. The image is doubled both in horizontal and vertical direction when the algorithm is processed once. Hence, the image can extend only by power of two along each direction.

Firstly, the $\mathbf{X}_{11}$ pixels of the LR image are directly written on the $\mathbf{Y}_{21,21}$ pixels of the HR images. After that, interpolation is 
processed in two steps. In the first step, we find the $\mathbf{Y}_{21+121+1}$

pixels from the $\mathbf{Y}_{21,21}$ pixels using Equation 2 [5].

$$
Y_{2 i+1,2 j+1}=\sum \sum \alpha_{2 k+l} \cdot Y_{2(i+k), 2(j+l)}
$$

The $\alpha$ vector in Equation 2 can be calculated as follow [5].

$$
\mathrm{C} \vec{\alpha}=\vec{y}
$$

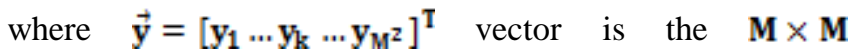
neighboring pixels of missing pixel which we want to interpolate them in diagonal axis. The matrix of $\mathrm{C}$ is the

$\mathbf{4} \times \mathbf{M}^{2}$ data matrix that contains 4 neighboring pixels of $\mathbf{y}_{\mathbf{k}}$ along diagonal direction in its kth column vector.

$\mathbf{Y}_{21+121+1}$ pixels can be found using $\alpha$ in Equation 2. In the second step, the remaining pixels are interpolated in the same way, but rotated by 45 degree.

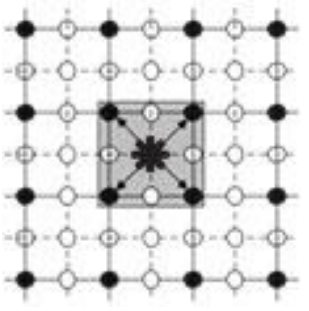

(a)

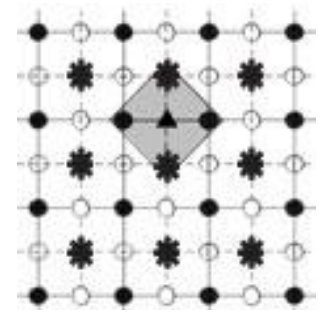

(b)
Fig.1: a) The pixel represented with asterix is the interpolated $Y_{2 i+12 j+1}$ pixel after first step. The black pixels, in the diagonal neighbors of pixel indicated by asterix, are the elements of the $y$ vector. b) The triangular pixel in the center of the window is interpolated in second step. The other pixels in the window are the elements of the $y$ vector rotated by 45 degree

\section{Cubic Spline}

Likewise the other interpolation techniques, the purpose of this interpolation technique is to obtain information about unknown points of a function using discrete points of the same function [6]. Generally, cubic spline functions are considered as interpolation over two nearest neighbors in each dimension. While spline functions are piecewise and continuous functions, cubic splines are piecewise, continuous and also third -order functions. Cubic spline functions should be symmetric around zero, and this is why we only consider the interval [0-2] to define the function. The general form of cubic spline function is given in Equation 4 [7].

$$
f(x) \begin{cases}a_{30} x^{3}+a_{20} x^{2}+a_{10} x+a_{00} & 0 \leq x \leq 1 \\ a_{31} x^{3}+a_{21} x^{2}+a_{11} x+a_{01} & 1 \leq x \leq 2\end{cases}
$$

Cubic spline functions have some constraints, when it is used for interpolation.

- Function's value should be ' 1 ' at point zero and should be ' 0 ' at point one and two.
- Function should be continuous at point zero and one.

- Function's slope should be ' 0 ' at point zero and two and the slope of the spline should be same approaching 1 and leaving 1.

Although there are three constraints above, the function has eight unknown values. Hence, a cubic spline function is determined depending on a constant value $a$ in Equation 5 [8].

$$
f(x) \begin{cases}(a+2) x^{3}-(a+3) x^{2}+1 & 0 \leq x \leq 1 \\ a x^{3}-5 a x^{2}+8 a x-4 a & 1 \leq x \leq 2\end{cases}
$$

In Cubic Spline method, when resampling of interpolating function, these four samples $f(d), f(1-d), f(1+d), f(2-d)$ are used, where $d$ is nearest point and it is in the interval [ $\left[\begin{array}{ll}0 & 1\end{array}\right]$. The sum of these four points should be equal to 1 [9].

In equation 4 , the function is positive in the interval $[0,1]$ and negative in the interval $[1,2]$ for a negative constant $a$. With the negative constant $a$, the function becomes a form of windowed sinc function that provides high frequency performance [7].

The constant $a$ is used as $a=-1, a=-0.75$, and $a=-0.5$. With $a=-0.5$ the interpolation function can exactly reconstruct any second degree polynomial and error approximates to zero as the third power of the sampling interval [8-10].

By choosing $a=-0.75$, the second derivatives of two cubic polynomials, (one is defined on the interval $[0,1]$, and the second one is defined on the interval $[1,2])$ is equal to each other at point 1[9].

When Rifmann chose $a=-1$, the functions' slope at point 1 match the sinc functions slope [9]-[10]. With $a=-1$, Cubic Spline functions becomes high resolution Cubic Spline functions, because it is resulted in some amplification of the frequencies and it can often be recognized by eye. Hence, this choose of $a=-1$ is used in image processing applications.

\section{EXPERIMENTS}

Firstly, LR reference images and its one pixel translated copy are obtained.

Step 1: Reference image is interpolated $N$ times by Bilinear, NEDI and Cubic Spline algorithm one by one.

Step 2: Interpolated new image is subsampled at every $N$ pixels in vertical and horizontal directions. After this process, $N$ copy of LR images, that have the same dimensions with LR reference image, is obtained.

Step 3: The correlations of every LR images, obtained in previous step, and one pixel translated images is computed.

Step 4: The LR image with the highest correlation value is found and the corresponding indices are multiplied by $1 / N$ to find the translational shift.

In the experimental studies, artificially created mean filter is used to deform $512 \times 512 \mathrm{HR}$ image for providing more quantitative evaluation. This image is then subsampled in every four pixels and $125 \times 125$ four images with 0.25 accuracy shifts are obtained. By this process we have equally deformed and shifted LR images. One of these images is chosen for reference image and another one is chosen for translated image 
that is utilized for computing the correlation in step four. The algorithm steps above are applied to reference image with one of these Bilinear, NEDI and Cubic Spline interpolation method at one time, and the amount of translation to define the registration parameters is computed. Projection onto Convex Sets (POCS) [11] method is applied to the image with the highest correlation for reconstructing it to the $512 \times 512$ pixels by utilizing the corresponding registration parameters which are obtained from the fourth step of our algorithm. This new super resolution image is compared to original $512 \times 512$ image using Peak Signal to Noise Ratio (PSNR) and Structural Similarity Index (SSIM) to evaluate the performance of each method.

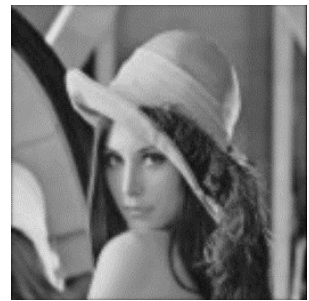

(a)

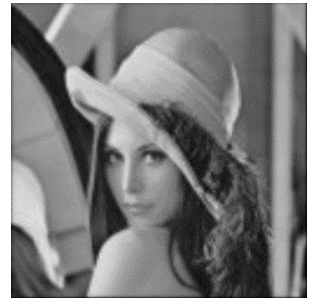

(c)

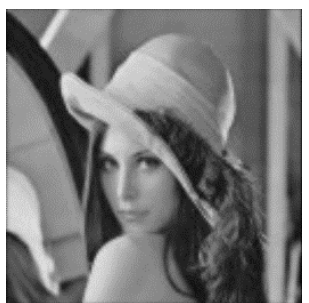

(b)

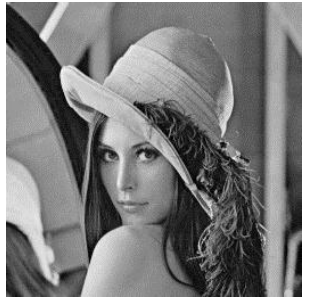

(d)
Fig. 2: Super resolution images reconstructed from Correlation combined with different Interpolation methods. a) Bilinear Interpolation and Correlation b) NEDI and Correlation c) Cubic Spline Interpolation and Correlation d) Original 512x512 image

TABLE I: PSNR RESULTS OF IMAGES CONSTRUCTED WITH BILINEAR, NEDI, SPLINE INTERPOLATION METHODS

\begin{tabular}{lccc}
\hline \hline Samples & Bilinear & NEDI & \multicolumn{1}{c}{ Spline } \\
\hline Lenna & 22.4262 & 22.7563 & $\mathbf{2 2 . 7 9 7 0}$ \\
Peppers & 22.1314 & 22.3743 & $\mathbf{2 2 . 6 2 6 5}$ \\
Barbara & 20.6139 & 20.7954 & $\mathbf{2 0 . 8 7 2 3}$ \\
Hill & 23.2043 & 23.4230 & $\mathbf{2 3 . 4 6 4 9}$ \\
\hline \hline
\end{tabular}

TABLE II: SSIM RESULTS OF IMAGES CONSTRUCTED WITH BILINEAR, NEDI, SPLINE INTERPOLATION METHODS

\begin{tabular}{lrrr}
\hline \hline Samples & Bilinear & NEDI & \multicolumn{1}{c}{ Spline } \\
\hline Lenna & 0.6764 & 0.6875 & $\mathbf{0 . 6 8 8 1}$ \\
Peppers & 0.7153 & 0.7255 & $\mathbf{0 . 7 2 8 4}$ \\
Barbara & 0.5295 & 0.5391 & $\mathbf{0 . 5 4 2 6}$ \\
Hill & 0.5337 & 0.5440 & $\mathbf{0 . 5 4 5 8}$ \\
\hline \hline
\end{tabular}

According to Table 1 and Table 2 Cubic Spline interpolation method produces better results compare to the other methods.

\section{CONCLUSION}

In this paper, registration is performed by utilizing different interpolation methods for evaluating the effect of sub pixel accuracy to the super resolution application. The proposed registration algorithm based on the principle of correlation maximization, used Bilinear, NEDI or Cubic Spline interpolation methods to provide sub pixel accuracy. Four images, Lenna Peppers, Barbara, Hill, are used to test these methods. All the test images are artificially deformed and the dimensions of these images subsampled to $125 \times 125$. The obtained results show that Cubic Spline interpolation algorithm combined with correlation technique presents higher performance for all images in the super resolution applications.

\section{REFERENCES}

[1] R. Y. Tsai, and T. S. Huang, "Multiframe image restoration and registration", in Advances in Computer Vision and Image Processing, T. S. Huang, Ed. JAI Press, 1984, Vol. 1, pp. 317-339.

[2] H. Cukur, H. Binol, F. S. Uslu, Y. Kalayc1, \& A. Bal, "Cross Correlation Based Clustering for Feature Selection in Hyperspectral Imagery," in Proc. 2015 9th International Conference on Electrical and Electronics Engineering (ELECO), 2015, pp. 232 - 236. http://dx.doi.org/10.1109/ELECO.2015.7394552

[3] H. Binol, A. Bal, and S. Dinc, "Classification on hyperspectral images using enhanced covariance descriptor," in Proc. Signal Processing and Communications Applications Conference (SIU), 2012 pp. 1- 4. http://dx.doi.org/10.1109/siu.2012.6204685

[4] F.S. Uslu, H. Binol, M. Ilarslan, A. Bal, "Improving SVDD classification performance on hyperspectral images via correlation based ensemble technique", Optics and Lasers in Engineering, (2016), http://dx.doi.org/10.1016/j.optlaseng.2016.03.006

[5] X. Li and M. T. Orchard, "New edge-directed interpolation", IEEE Trans. Image Processing, vol. 10, pp. 1521-1527, 2001. http://dx.doi.org/10.1109/83.951537

[6] Wikipedia contributors. (March 2016). Spline interpolation. Wikipedia, The Free Encyclopedia [Online]. Available: https://en.wikipedia.org/w/index.php?title=Spline_interpolation\&oldid= 709116406.

[7] J.A. Parker, R.V. Kenyon, and D.E. Troxel, "Comparison of Interpolating Methods for Image Resampling", IEEE Transactions on Medical Imaging, vol. 2, pp. 31-39, 1983. http://dx.doi.org/10.1109/TMI.1983.4307610

[8] R. G. Keys, "Cubic convolution interpolation for digital image Processing," IEEE Trans. Acoust., Speech, Signal Processing, vol. ASSP-29, pp. 1153-1160, 1981. http://dx.doi.org/10.1109/TASSP.1981.1163711

[9] K. W. Simon, "Digital image reconstruction and resampling for geometric manipulation," in Proc. IEEE Symp. Machine Processing of Remotely Sensed Data, 1975, pp. 3A- 1-3A- 11.

[10] S. S. Rifman, "Digital rectification of ERTS multispectral imagery," in Proc. Symp. Significant Results Obtained from the Earth Resources Technology Satellite-i, 1973, vol. 1, sec. B NASA SP-327, pp. 11311142.

[11] A.M. Tekalp, M.K. Ozkan, and M.I. Sezan, "High-resolution image reconstruction from lower-resolution image sequences and space varying image restoration,"in Proc. IEEE Int. Conf. Acoustics, Speech and Signal Processing (ICASSP), 1992, vol. 3, pp.169-172. http://dx.doi.org/10.1109/icassp.1992.226249 